
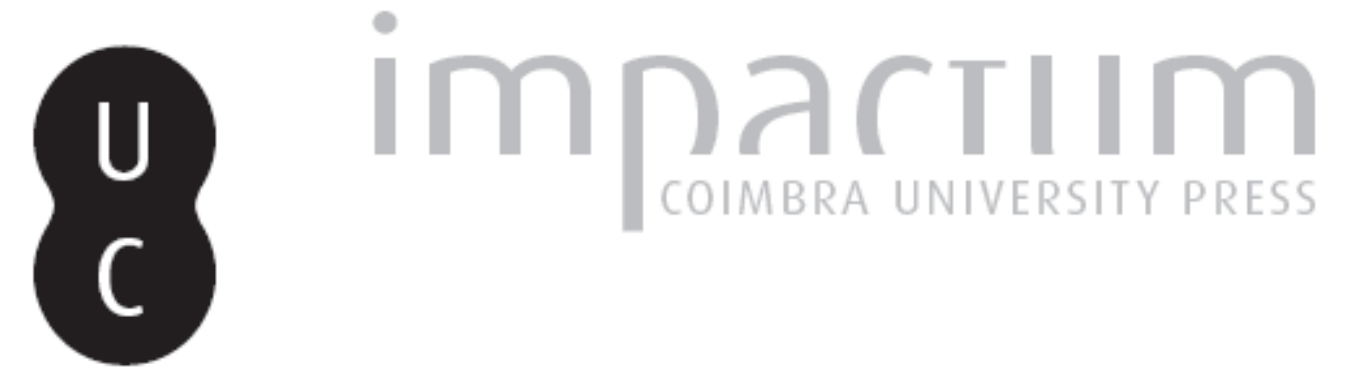

La poesia come antologia: il Novecento portoghese nella tradizione traduttiva italiana Autor(es): $\quad$ Russo, Vincenzo

Publicado por: Imprensa da Universidade de Coimbra

URL persistente:

URI:http://hdl.handle.net/10316.2/42500

DOI:

DOI:https://doi.org/10.14195/0870-8584_7_7

Accessed : $\quad$ 26-Apr-2023 11:06:26

A navegação consulta e descarregamento dos títulos inseridos nas Bibliotecas Digitais UC Digitalis, UC Pombalina e UC Impactum, pressupõem a aceitação plena e sem reservas dos Termos e Condições de Uso destas Bibliotecas Digitais, disponíveis em https://digitalis.uc.pt/pt-pt/termos.

Conforme exposto nos referidos Termos e Condições de Uso, o descarregamento de títulos de acesso restrito requer uma licença válida de autorização devendo o utilizador aceder ao(s) documento(s) a partir de um endereço de IP da instituição detentora da supramencionada licença.

Ao utilizador é apenas permitido o descarregamento para uso pessoal, pelo que o emprego do(s) título(s) descarregado(s) para outro fim, designadamente comercial, carece de autorização do respetivo autor ou editor da obra.

Na medida em que todas as obras da UC Digitalis se encontram protegidas pelo Código do Direito de Autor e Direitos Conexos e demais legislação aplicável, toda a cópia, parcial ou total, deste documento, nos casos em que é legalmente admitida, deverá conter ou fazer-se acompanhar por este aviso. 


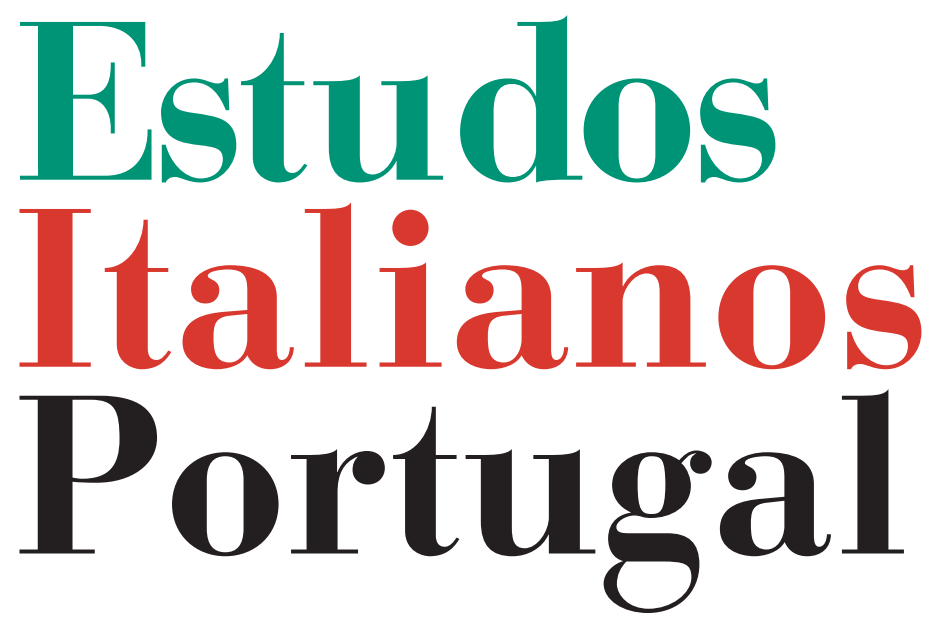

Instituto

Italiano

de Cultura

de Lisboa

Nova Série

$\mathbf{N}^{\mathbf{0}} 7$

2012 


\section{LA POESIA COME ANTOLOGIA: \\ IL NOVECENTO PORTOGHESE NELLA TRADIZIONE TRADUTTIVA ITALIANA}

VINCENZO RUSSO^

Las antologias son mapas, no territorios.

Andrés Sánchez Robayna

\section{QUESTIONI PRELIMINARI}

Un giorno, prima o poi, quando qualcuno scriverà la storia della ricezione traduttiva italiana delle lettere portoghesi, analizzando in particolare la costellazione poetica novecentesca, non potrà esimersi dal confrontarsi con una stratificata tradizione antologica. La poesia in traduzione, soprattutto nel Novecento, è stata diffusamente veicolata attraverso la forma antologica per varie e svariate ragioni, non ultima quella per cui il genere lirico si adatta meglio al modello antologico: il che è tanto più vero per le antologie non esclusivamente monoautoriali ma anche per quelle collettive che definiamo, sulla scorta di un'abbondante riflessione critica, panoramiche o programmatiche. Anche il caso della poesia portoghese del Novecento tradotta in italiano non fa eccezione. Le antologie collettive di poesia portoghese in italiano,

* Laurea in Lettere Moderne (1998) e dottorato in Letteratura Portoghese (2003) presso l'Università di Bologna.

Professore di Letteratura Portoghese e Letteratura Brasiliana presso l'Università di Milano. Si dedica allo studio della poesia portoghese moderna e contemporanea, del pensiero portoghese e degli immaginari imperiali africani dell'Ottocento e del Novecento. Ha pubblicato in volume Tenebre Bianche. Immaginari coloniali "fin-de-siècle" (2008) e Suspeita do Avesso. Barroco e Neo-Barroco na Poesia Portuguesa Contemporânea (2008). 
ma in genere in lingua "straniera", non contribuiscono a migliorare o a sublimare la qualità dei testi di origine, piuttosto ne arricchiscono la storia della loro trasmissione ${ }^{1}$. Senza voler riprendere qui l'intero dibattito critico sulla forma della antologia che come ricorda Carlo Ossola "è stata poco studiata come meccanismo specifico, interessando in genere di più $i$ selezionati (o polemicamente gli esclusi) che i meccanismi costitutivi della selezione, cioè della formulazione dell'area ripetibile, citabile, e memorabile, che consolida il "letterario" come genere "normato" tra i documenti della scrittura" 2 , bisogna tuttavia riconoscere come la critica delle antologie poetiche abbia fatto spesso ricorso a strategie retoriche che hanno trasformato il discorso critico in una vera e propria tautologia: l'antologia intesa come teologia di salvezza riprodurrebbe l'antico schema di canzoniere di assenze e presenze, dove gratificazioni o esclusioni sono gli unici elementi che stimolano il dibattito ${ }^{3}$, oppure l'idea rigida di interpretare l'antologia come super-categoria non passibile di sfumature o distinzioni, ma solo come prodotto di deliberate opzioni estetiche, oppure ancora la tendenza (pericolosa) ma consolidata di considerare l'antologia qualcosa d'altro (manuale, storia letteraria, saggio, etc.) rispetto a ciò che essa a livello di genere testuale è. Al di là, dunque, di ogni considerazione che si focalizzi sull'antologia come genere dell'elezione (di quel testo tra tanti possibili), non appare scontato insistere su di essa come "genere della ripetizione (di un testo-matrice,

${ }^{1}$ Sulla stratificata questione del rapporto fra Canone letterario e antologia si legga almeno il capitolo "Antología y canon literario", J. F. Ruiz Casanova, Anthologos: Poética de la antología poética, Madrid, Cátedra, 2007, pp. 141-160.

${ }^{2}$ C. Ossola, "Antologia come ontologia", Brano a brano. L'antologia di italiano nella scuola media inferiore, a cura di C. Ossola, Bologna, Il Mulino, 1978.

3 "Parece, pues, una paradoja que el destino de libros tan gruesos se decida en sus últimas paginas" scrive non senza ironia José Angel Cilleruelo, "Reseña de Los versos satíricos de Eduardo Moga”, El Ciervo, 612 (marzo de 2002), <www.elciervo.es>, p. 46. 
ridotto a brani, e sommato)" ${ }^{4}$ : se, infatti, come luogo della citazione, ogni selezione antologica "è sempre [...] una selezione di secondo grado: opera, cioè, su materiali già selezionati" ${ }^{5}$ a cui il processo elettivo conferisce esemplarità perché insiste sulla ripetizione del medesimo, è vero anche che tale selezione codificando il parziale come ripetibile fa sì che si possa distinguere nel testo d'origine (l'opera completa di un autore, ammettiamo) "una parte da citare come memorabile e una parte da omettere come secondaria, una parte da reduplicare e una da assimilare alla prima, un testo insomma e un resto"6.

Prima di addentrarci nell'analisi di quel segmento ben specifico della tradizione traduttiva italiana, possiamo riconoscere alcuni nodi critici che dovranno necessariamente essere presi in considerazione anche solo come promemoria per una futura storia della ricezione della Letteratura Portoghese in Italia. 1. Uno studio, dall'ampio spettro disciplinare, delle relazioni culturali fra Italia e Portogallo nel Novecento non dovrà trascurare un censimento sistematico dei rapporti istituzionali (diplomazie, enti, istituti) fra i due paesi; 2 . Una storia delle relazioni accademiche - segnata simbolicamente dalla creazione della prima cattedra di Letteratura Portoghese in Italia istituita a Napoli nel 1956 a opera di Giuseppe Carlo Rossi ${ }^{7}$ il primo lusitanista accademico - contribuirebbe a fornire non solo un documento di archeologia scientifica

${ }^{4}$ C. Ossola, op. cit., p. 12.

${ }^{5}$ A. Quondam, "Ideologia e struttura della forma antologia", in Petrarchismo mediato. Per una critica della forma "antologia", Roma, Bulzoni, 1974, p.15.

${ }^{6}$ C. Ossola, cit., p. 18.

${ }^{7}$ Benché esistessero già corsi liberi di letteratura portoghese (uno di questi fu tenuto a Roma negli anni Venti da Mario Pelaez), Giuseppe Carlo Rossi viene considerato il primo lusitanista accademico in Italia in quanto ricopre la carica di libero docente di lingua e letteratura portoghese dal 1942. Incaricato di tale disciplina all'Università di Roma (1948-1957), e ordinario nella prima cattedra italiana della materia (1956), presso l'Istituto Universitario Orientale di Napoli. 
e erudita ma ridirebbe, sul versante della tradizione traduttiva, il complesso rapporto fra i traduttori dal portoghese accademici e quelli non accademici. Che la questione sia dirimente è confermato dal fatto che la storia della ricezione letteraria portoghese in Italia, e in particolare la ricezione poetica, conosca un mutamento di paradigma proprio a partire dal secondo dopo-guerra, quando con la diffusione dell'insegnamento universitario della Lingua e della Letteratura Portoghese, si forma una costellazione di accademici portoghesisti che si dedicherà alla divulgazione e alla diffusione di quella letteratura attraverso un ingente sforzo traduttivo. Per limitarci alla sola ricezione poetica del Portogallo novecentesco, possiamo dunque affermare che se, da un lato, il canone traduttivo della prima metà del secolo è codificato, come è normale che avvenga, non da specialisti ma da figure ecclettiche come il genovese Guido Battelli - lettore di italiano a Coimbra nel 1930 e amico-corrispondente della poetessa Florbela Espanca, curatore di un pioniere ma controverso volume Lirici Portoghesi Moderni del $1929^{8}$ - o dal diplomatico Enzio di Poppa Vólture', curatore, tra le altre cose, di Poeti moderni portoghesi del 1942, dall'altro, la poesia portoghese moderna e contemporanea, soprattutto nella

${ }^{8}$ L'antologia Lirici Portoghesi Moderni uscita per l'Editore Carabba di Lanciano - unica casa editrice italiana nella prima metà del Novecento a promuovere un coerente progetto editoriale, oggi perfino sorprendente, sulla letteratura portoghese - ha sempre sorpreso per la selezione degli autori inclusi (da Garrett fino ai saudosistas e ai neoromantici di inizio secolo) e per le esclusioni eccellenti (i modernistas).

${ }^{9}$ E' più che probabile che la primissima traduzione di un testo di Fernando Pessoa comparsa in Italia fosse la traduzione di Enzio di Poppa Vólture in Poeti moderni portoghesi, Lisbona, Edizioni di Estudos Italianos em Portugal, 1942, nello stesso anno in cui si iniziano a pubblicare in Portogallo le Obras Completas de Fernando Pessoa per i tipi dell'Ática. L'introduzione firmata da Gino Saviotti ha per titolo: "Presentazione (agli italiani) dei moderni poeti portoghesi”. Il volume sceglie le principali figure del movimento del modernismo portoghese e, tra gli autori, compare anche Fernando Pessoa con i componimenti Il guardiano di greggi, Passi della croce, Tutta notte non venne il sonno, Chi batte alla mia porta. Inoltre compaiono anche Appunto di Álvaro 
forma di antologia collettiva, è stata introdotta in Italia quasi esclusivamente da traduttori accademici, come lo sono la maggioranza dei curatori-traduttori (solo in un caso il curatore non coincide con il traduttore ${ }^{10}$ ) delle cinque antologie che abbiamo deciso di prendere in esame.

\section{IL NOVECENTO PORTOGHESE IN VERSI ITALIANI}

Abbiamo optato per restringere l'analisi della ricezione poetica del Novecento Portoghese in forma antologica a cinque case studies (apparsi tra il 1971 e il 2003) che rappresentano, nella loro individualità, un paradigma editoriale per comprendere il processo complesso e stratificato di ricezione degli ultimi quarant'anni di Letteratura Portoghese in Italia e al contempo un utile laboratorio di disamina critica di certe questioni intimamente legate alla forma dell' antologia (poetica dell' antologia poetica, la sua tipologia, il rapporto con il Canone letterario, i meccanismi di selezione, la figura dell'antologista).

a. La parola interdetta. Poeti surrealisti portoghesi, a cura di Antonio Tabucchi, Torino, Einaudi, 1971.

b. Da Pessoa a Oliveira. La moderna poesia portoghese. Modernismo, Surrealismo, Neorealismo, a cura di Giuseppe Tavani, Milano, Accademia, 1973.

c. La Nuova Poesia Portoghese, a cura di Carlo Vittorio Cattaneo, Roma, Edizioni Abete, 1975.

d. Poeti Portoghesi Contemporanei, a cura di Manuel G. Simôes, traduzioni di Giampaolo Tonini, Venezia, Centro Internazionale della Grafica, 1999.

de Campos e le Odi I, V, IX di Ricardo Reis. Saviotti parla di "pseudonimi” e di una possibile filiera letteraria della poetica di Pessoa che, a mo' di ipotesi, includerebbe certi tocchi caratteristici dei "versetti gozzaniani", influssi del futurismo e dei suoi sviluppi per poi approdare come tappa finale al classicismo formale delle Odi di Reis.

${ }^{10}$ Il solo Carlo Vittorio Cattaneo, figura di pubblicista e di divulgatore soprattutto della poesia portoghese della seconda metà del Novecento in Italia, può essere considerato un traduttore non accademico. 
e. Inchiostro nero sulla carta che danza. Antologia di poesia portoghese contemporanea, a cura di Giulia Lanciani, Milano, Mondadori, $2003^{11}$.

Le cinque antologie, pur con tutte le differenze critiche e selettive del caso (l'inevitabile sovrapponibilità di certi autori, la dichiarata filiazione dell'una rispetto all'altra almeno in termini cronologici ${ }^{12}$, etc), si costituiscono, per proprio

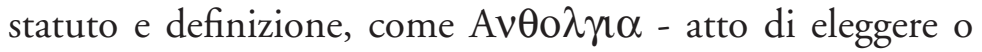
selezionare fiori - di un corpus testuale esclusivo e ristretto ${ }^{13}$ : la poesia portoghese del Novecento che viene cronologicamente incastonata fra le Impressóes do Crepúscolo (1913) di Fernando Pessoa come limite basso (Tavani, 1973) e i testi di Nossa Senhora das Tempestades di Manuel Alegre pubblicati già nel 2001 come limite alto (Lanciani, 2003). In vero, pur oscillando nella titolazione ("Poeti Portoghesi Contemporanei", "Antologia di poesia portoghese contemporanea", etc.), le cinque antologie sono tutte accomunate dal fatto di presentarsi come silloge, selezione non di testi, ma di poeti. Una

${ }^{11}$ Questo volume ha un importante precedente nell'articolo (con testo introduttivo e traduzione di testi poetici) di Giulia Lanciani "Poeti portoghesi del Novecento" uscito nel primo numero della rivista Trame di Letteratura Comparata (2000), ora consultabile qui: <http://www.dllc.unicas.it/riviste/trame/trame_vol1/lancianil.pdf>.

12 "Il lettore italiano ha già a disposizione un'antologia della moderna poesia portoghese, di cui il presente lavoro potrebbe essere considerato la prosecuzione fino ai nostri giorni. Va però detto che la limitazione anagrafica lascia scoperta una zona non inclusa nel libro di Tavani e che avrebbe avuto qui, per ragioni di poetica, la sua giusta collocazione. Si tratta di poeti affermatisi intorno al 1956, ma nati prima del 1930 (basterà citare quattro nomi: Fernando Echevarria, Fernando Guimarães, Jorge de Amorim e João Rui de Sousa). La loro inclusione avrebbe aumentato di molto le presenze e, conseguentemente, avrebbe troppo limitato il numero dei testi per singolo autore. Ed è mia convinzione che presentare per la prima volta a un pubblico straniero un poeta, esemplificandolo con quattro o cinque composizioni, sia un'operazione culturale di nessun efficacia" scrive Carlo Vittorio Cattaneo, "Introduzione", La Nuova Poesia Portoghese, Roma, Edizioni Abete, 1975, p. VII.

${ }^{13}$ Si veda alla fine la tabella con alcuni dati statistici relativi alle cinque antologie. 
costellazione dunque coerente di antologie che ci ha permesso di tralasciare tutti quei volumi che pur selezionando testi poetici del Novecento portoghese si fondano su criteri antologici diversi: l'antologia generale dell'intera storia della poesia portoghese come quella tentata da Luciana Stegagno Picchio nella collana "La biblioteca di Repubblica" (volume 16, 2004) che, tra l'altro, pur titolandosi Antologia della poesia portoghese e brasiliana presentava anche una sezione dedicata ai testi poetici delle letterature dei paesi dell'Africa lusofona, l'antologia panoramica e senza restrizione di genere testuale come le classiche Pagine della Letteratura Portoghese, a cura di P. A. Jannini, Nuova Accademia Editrice, Milano, 1955 oppure l'antologia pedagogico-didattica pubblicata da G. Ricciardi- R. Barchiesi (Antologia della letteratura portoghese : testi e traduzioni, Tullio Pironti, Napoli, 1998) ${ }^{14}$; e poi le antologie "settoriali" come quella che spazia lungo l'intera storia poetica nazionale ma selezionando un genere lirico preciso come il sonetto ${ }^{15}$, come quella di poesia portoghese contemporanea femminile ${ }^{16}$ o geograficamente limitata agli autori di Madera ${ }^{17}$. Abbiamo inoltre tralasciato una recente antologia di poesia contemporanea che potremmo definire plurilinguistica (per la presenza di autori portoghesi e gali-

${ }^{14}$ Sulla questione della antologia pedagogica o teaching anthology in cui la sua funzione informativa e estetica si conforma su una determinata intenzionalità storica dell'antologista si veda almeno Glen M. Johnson, "The Teaching Anthology and the Canon of American Literature: some notes on Theory and Practice", in V. Nemoianu and R. Royal eds., The Hospitable Canon. Essays on Literary Play, Scholarly Choice and Popular Pressures, Philadelphia-Amsterdam, John Benjamins Pub. Co., 1991 e D. Wu, "Editing Student Anthologies: The Burning Question" < http://www.erudit.org/ revue/ron/1997/v/n7/005757ar.html $>$.

${ }^{15}$ Il sonetto portoghese, a cura di Leo Negrelli, Firenze, Il Fauno, 1964.

${ }^{16}$ Gli abbracci feriti: poetesse portoghesi di oggi, a cura di Adelina Aletti, introduzione di Luciana Stegagno Picchio, Milano, Feltrinelli, 1980.

${ }_{17}$ Poeti contemporanei dell'Isola di Madera, a cura di Giampaolo Tonini, trad. Giampaolo Tonini, Venezia, Centro Internazionale della Grafica, 2001. 
ziani) licenziata da Alessandro Agostinelli e Manuele Masi$\mathrm{ni}^{18}$.

\section{Poetica dell'antologie POetiche}

Muovendo da una proposta tipologica di antologie poetiche fondata su un dualismo che già Menéndez Pelayo difendeva, il critico spagnolo José Francisco Ruiz Casanova classifica le antologie di poesia in lingua spagnola del Novecento in due grandi gruppi (panoramiche e programmatiche, a loro volta suddivise in sottogruppi) riconoscendo tuttavia il carattere poroso e non totalmente escludente di questi insiemi. Pur evitando di applicare meccanicamente questa classificazione alle nostre cinque antologie, è indubbio che l'antologia tabucchiana può essere inclusa fra le antologie programmatiche di gruppo che "forman un conjunto más o menos coherente de escritores coincidentes, siempre desde un punto de vista amplio, en un marco temporal concreto" 19 e che soprattutto abbia l'esigenza di "cohesionar una estetica que se alza con rasgos diferenciados de los autores de más edad" 20 . In effetti, se il corpus selezionato di poeti non ha bisogno di esser giustificato dal momento che l'(est) etica surrealista ne legittima o legittimerebbe ${ }^{21}$ la coesione imponendo l'esemplarità di certi autori e certi testi, lo stu-

18 Poetica Atlantica 2007, Pisa, ETS, 2008. Da segnalare, a mo' di curiosità, l'antologia curata da Prospero Peragallo, alla fine dell'Ottocento, che si può considerare un illustre antenato di antologia plurilingue Mazzolini di poesie portoghesi e sivigliane (1898) che includeva tra gli altri testi di Camôes, Antero de Quental, Castilho, Teófilo Braga e Almeida Garrett. Su Prospero Peragallo "un vero agente culturale fra Italia e Portogallo alla fine dell'800 e inizi del 900", si legga Cristina Rosa, "Prospero Peragallo, un agente culturale fra Italia e Portogallo: una bio-bibliografia”, Amicitiae Munus, a cura di G. Sommariva, La Spezia, Agorà Edizioni, 2006.

${ }^{19}$ E. Bayo, La poesia española en sus antologías (1939-1980), Lleida, Pagès EditorsUniv. De Lleida, 1994, 2 vols, p. 45.

${ }^{20}$ J. F. Ruiz Casanova, cit., p. 135.

${ }^{21}$ Non poche polemiche suscitò, anche in Portogallo, la presenza fra i surrealisti tout court di un autore come Herberto Helder. 
dio introduttivo diviso in sette capitoli funziona da paratesto preparatorio a un'antologia del surrealismo fra i molti surrealismi portoghesi e possibili. Del resto, come viene ricordato da Ruiz Casanova:

Una antologia programática tiende a ser una muestra y, como tal, no da cuenta de generaciones completas, ni de épocas, sino de aquellos autores que a juicio del antólogo mejor representan los presupuestos teóricos y crítico de su antologia 22 .

Quanto alle altre quattro antologie, avremo bisogno di analizzare, caso per caso, la loro specificità che ci rivelerà quanto scivolosa e problematica sia l'applicazione di certe tipologie preconfezionate.

Nel caso di Tavani (1973), la definizione di antologia panoramica de época non è sufficiente a tradurre il criterio che sottace alla selezione degli autori che sono ordinati in base a un criterio che non è puramente storico bensì estetico. Proprio il criterio estetico rende Da Pessoa a Oliveira (se fosse cronologico avrebbe dovuto titolarsi Da Afonso Duarte a Luiza Neto Jorge) un testo selettivo-elettivo che privilegia, a mo' di contenitore programmatico, le tre grandi dorsali esteticoletterarie della prima metà del Ventesimo secolo (modernismo - neorealismo - surrealismo, come recita il sottotitolo) le quali se, da un lato, hanno il pregio di semplificare (ancorché livellando certi processi poetici "recalcitranti" a questa tentazione sistematica) la modernità poetica novecentesca, dall'altro non esauriscono il portato teorico delle poetiche moderne in Portogallo ${ }^{23}$.

22 J. F. Ruiz Casanova, cit., p. 167.

${ }^{23}$ Come del resto mostra l'inclusione di poeti, giusto per esemplificare, come Manuel Alegre che oggi si iscrive pienamente in quella corrente che è definibile come "neo neorealista" che debutta negli Anni Sessanta o come Luiza Neto Jorge esponente di una delle ultime avanguardie poetiche nazionali, quella Poesia 61 che insiste teoricamente per una rivalorizzazione del linguaggio nella prassi poetica. 
Il volume di Cattaneo (1975) - pur reclamandosi come "prosecuzione fino ai nostri giorni" di Tavani (1973) - dichiara sin da subito la sua vocazione antologica generazionale, stabilendo come criterio di inclusione il limite cronologico dell'anno 1930, anno di nascita di Herberto Helder eletto da Cattaneo come punto di partenza e "autore-ponte". La composita generazione degli Anni Sessanta è egemonizzata dalla presenza degli esponenti (quattro su cinque) di Poesia 61, mentre i due giganti della seconda metà del Novecento anche in virtù degli esiti successivi della poesia nazionale lo stesso Herberto e Ruy Belo - sono affiancati dai nomi di Pedro Tamen (appartenente a quel gruppo di virtuosisti "neobarocchi" già attivo alla fine degli Anni Cinquanta) e di Manuel Alegre, sineddoche di una tendenza poetica in cui la resa realista si intreccia con le istanze politiche di lotta al regime e alla guerra coloniale. La generazione degli Anni Settanta, pur difficilmente storicizzabile nel 1975, è contrassegnata dalla presenza di un buon numero (cinque) di poeti che forse con l'eccezione di Nuno Guimarães, appartengono oggi di diritto al Canone della poesia novecentesca. Antologia programmatica di generazione ("i poeti qui rappresentati costituiscono soltanto le "forze nuove" apparse in Portogallo negli ultimi 15-18 anni"24), il libro di Cattaneo (1975) mostra il suo deficit metodologico allorché vuole mascherare una certa unilateralità di intenzioni estetiche sotto una rigorosa continuità storica, come risulta evidente nel caso dell'esclusione della Poesia Sperimentale:

L'esclusione da questa antologia dei poeti sperimentali (sia gli epigoni del concretismo brasiliano, sia quelli che hanno battuto vie più personali) è dovuta esclusivamente a limiti di spazio e alla difficoltà (spesso impossibilità) di traduzione $e^{25}$.

${ }^{24}$ C.V. Cattaneo, "Introduzione", p. VIII.

${ }^{25} \mathrm{Ib}$. Che la retorica del limite imposto o autoimposto all'antologista faccia parte della costellazione discorsiva prologale è stato ampiamente dimostrato. 
Come Tabucchi (1971) e Cattaneo (1975), anche il volume di Simões (1999) - pensato e pubblicato però in un contesto storico-culturale (doppio) portoghese e italiano totalmente differente rispetto ai primi - è un'antologia di poesia postpessoana che ammette inesorabilmente il grado di ambiguità e di convenzionalità di un termine come contemporaneo:

Che cosa si intende per "poesia contemporanea" e quali sono le frontiere temporali che segnano i limiti dell'oggetto circoscritto? Si sa che il termine "contemporaneo", anche a livello di prodotto letterario, comporta una certa elasticità soprattutto quando si considera il periodo più recente, suscettibile com'è di aggiustamenti per forza del lento lavorio di sedimentazione al quale va ovviamente soggetto l'opera di ciascun scrittore, poeta o romanziere che $\operatorname{sia}^{26}$.

Il criterio adottato di selezione, sin da subito esplicitato nell'Introduzione, è una mediazione fra limiti cronologici e gruppi generazionali rappresentati ${ }^{27}$ : le generazioni degli Anni Cinquanta e Sessanta, almeno così come codificate dalla storiografia critica portoghese, sono quantitativamente le più rappresentate (rispettivamente 6 e 5 poeti), incorniciate dall'eccezione Sophia e Eugénio de Andrade (rappresentanti del gruppo post secondo Modernismo di Cadernos de Poesia) e da Vasco Graça Moura e António Franco Alexandre, oggi, solitamente raccolti sotto l'ombrello critico di quel regresso ao real proprio di certe tendenze degli Anni Settanta.

${ }^{26}$ M. G. Simões, "La poesia portoghese contemporanea", Poeti Portoghesi Contemporanei, a cura di Manuel G. Simões, traduzioni di Giampaolo Tonini, Venezia, Centro Internazionale della Grafica, Venezia, p. 8.

27 "In questo modo, dopo una lunga e meticolosa operazione di analisi dell'intero corpus, il criterio è stato quello di scegliere i poeti più rappresentativi, vivi e attivi, che hanno pubblicato l'opera prima negli anni Cinquanta e Sessanta, con due sole eccezioni che concernono i casi di Sophia de Mello Breyner e di Eugénio de Andrade, già apparsi nel decennio precedente. Sono però due nomi che nessuna antologia poetica del Novecento avrebbe potuto dimenticare, dato che si tratta di due voci di estrema importanza quando consideriamo la poesia portoghese del nostro secolo", scrive M. G. Simões, cit., p. 8. 
Più complessa è l'articolazione discorsiva che sottace alla Premessa di Lanciani (2003) da cui possiamo far emergere almeno due questioni critiche relative alla specifica poetica dell'antologia che essa racchiude. Se solitamente l'avantesto (prologo, premessa, introduzione) di ogni antologia si propone un doppio fine - presentare le opere e gli autori e giustificarne la loro presenza, cioè, il processo selettivo - nella Premessa di Lanciani (2003) si lascia il compito di presentare la cartografia lacunosa di autori al testo introduttivo di Manuel Gusmão "Cinque partiture, cinque voci. Differenze erranti" ${ }^{28}$ per indugiare invece sul senso politico del processo selettivo, intendendo qui politico come opera di negoziazione, di un patto tra il tempo di scrittura, il tempo di lettura e il tempo di rilettura, e una posizione estetica e storiografica che l'antologista vuole mostrare e difendere in vista dell'unità del libro. Lungi dalla pretesa di fotografare per sineddoche la poesia portoghese contemporanea (il cui limite a quo è il 1956 mentre quello ad quem è il 2001), il momento elettivo effettuato dopo una selezione della selezione ("Del resto, è in questa prospettiva che è avvenuta la selezione: nel corso e attraverso la traduzione - inizialmente non limitata ai soli autori e ai soli testi qui pubblicati - il materiale da privilegiare si è andato via via affermando con una propria forza intrinseca quasi indipendente dal mio gusto personale, suggerendo e anzi decretando esso stesso linee, strutture e limiti del prodotto finale"29) appare come una mediazione fra un elemento via via evanescente come le scelte personali dell'antologista e l'imposizione quasi per forza propria di al-

${ }^{28}$ Oltre al testo introduttivo del docente e critico portoghese, l'antologia è corredata per ciascuno dei cinque autori di un profilo biobibliografico (presente, del resto, anche nelle altre antologie poetiche studiate) e da un testo breve di poetica esplicita a firma dello stesso autore.

${ }^{29}$ G. Lanciani, "Premessa”, Inchiostro nero sulla carta che danza. Antologia di poesia portoghese contemporanea, a cura di Giulia Lanciani, Milano, Mondadori, 2003, pp. V-VI. 
cuni testi e alcuni autori ("soverchiando quasi la volontà di chi avrebbe dovuto invece procedere ex oficio a inserimenti e esclusioni”30). La rivendicazione di una dissolvenza da parte dell'antologista in funzione dei "condizionamenti emanati dal processo [selettivo] stesso" 31 non fa però che riecheggiare una presenza come quella dell'antologista che in quanto autore vero e proprio dà sempre il senso e la forma alla sua antologia poetica, scandisce all'origine i presupposti teorici della sua poetica. Se allora nell'antologista italiano di poesia portoghese del Novecento si intersecano la figura del traduttore-curatore e quella dell'accademico, conferendo al suo status un sovrappiù di responsabilità scientifica e culturale, il richiamo a qualsivoglia invisibilità oltre che utopico sembra davvero poco desiderabile.

L'invito, insomma a mo' di conclusione, è quello di ripensare l'antologia italiana di poesia portoghese del Novecento come frutto stratificato di un fascio spesso inestricabile, di tensioni, di desideri, di opzioni estetiche e storiografiche, di gusti personali e contingenze temporali, dove una certa memoria poetica del passato continua costantemente a fare $\mathrm{i}$ conti con la critica del tempo presente. L'antologia, come ogni archivio (non solo testuale), deve combattere la sua lotta per la gestione della memoria. Una memoria che, ancorché residuale, può provare a resistere alla consunzione del tempo degli uomini.

\footnotetext{
${ }^{30} \mathrm{Ib}$.

${ }^{31} \mathrm{Ib}$.
} 
Lo schema riproduce la presenza degli autori per antologia e il numero - tra parentesi - di testi poetici selezionati per ogni autore. Le cinque antologie, senza contare i "doppioni", includono un numero di 53 poeti compresi per anno di nascita fra il 1884 (Afonso Duarte) e il 1949 (Nuno Júdice).

\begin{tabular}{|c|c|c|c|c|}
\hline TABUCCHI (1971) & TAVANI (1973) & CATTANEO (1975) & SIMŌES (1999) & LANCIANI (2003) \\
\hline M. Cesariny (22) & F. Pessoa (7) & H. Helder (11) & $\begin{array}{l}\text { S. de Mello Breyner } \\
\text { Andresen (11) }\end{array}$ & P. Tamen (34) \\
\hline A. O’Neill (16) & M. de Sá Carneiro (5) & R. Belo (12) & E. Gonçalves (5) & G. Cruz (31) \\
\hline A. M. Lisboa (15) & $\begin{array}{l}\text { J. de Almada } \\
\text { Negreiros (3) }\end{array}$ & P. Tamen (15) & E. de Andrade (10) & V. Graça Moura (8) \\
\hline F. Alves dos Santos (2) & A.Côrtes-Rodrigues(2) & M. Alegre (6) & A. Ramos Rosa (6) & M. Alegre (12) \\
\hline H. Helder (1) & A. Botto (2) & A. Silva Carvalho (5) & F. Guimarães (7) & N. Júdice (32) \\
\hline \multirow[t]{29}{*}{ Cruzeiro Seixas (4) } & Duarte (3) & $\begin{array}{l}\text { F. Hasse Pais } \\
\text { Brandão (11) }\end{array}$ & F. Echevarría (6) & \\
\hline & A. de Sousa (2) & L. Neto Jorge (12) & A. Hatherly (4) & \\
\hline & S. Dias (3) & G. Cruz (19) & H. Helder (3) & \\
\hline & J. Régio (3) & N. Guimarães (5) & P. Tamen (7) & \\
\hline & M. Torga (6) & $\begin{array}{l}\text { J. M. Fernandes } \\
\text { Jorge (15) }\end{array}$ & M. Alegre (7) & \\
\hline & Casais Monteiro (2) & A.F. Alexandre (2) & C. de Brito (5) & \\
\hline & A. Pedro (3) & J.M. Magalhães (11) & $\begin{array}{l}\text { F. Hasse Pais } \\
\text { Brandão (6) }\end{array}$ & \\
\hline & M. Cesariny (1) & N. Júdice (10) & A.Silva Carvalho (6) & \\
\hline & A. M. Lisboa (5) & & G. Cruz (8) & \\
\hline & A. O’Neill (6) & & V. Graça Moura (4) & \\
\hline & F. Namora (1) & & A.F. Alexandre (6) & \\
\hline & M. Dionísio (4) & & & \\
\hline & C. de Oliveira（5) & & & \\
\hline & J.J. Cochofel (7) & & & \\
\hline & J. Namorado (2) & & & \\
\hline & M. da Fonseca (2) & & & \\
\hline & Feijó (2) & & & \\
\hline & J.Gomes Ferreira (9) & & & \\
\hline & Rodrigues (5) & & & \\
\hline & S. M. B. Andresen (7) & & & \\
\hline & E. de Andrade (5) & & & \\
\hline & J. de Sena (4) & & & \\
\hline & Ramos Rosa (3) & & & \\
\hline & E. Gonçalves (4) & & & \\
\hline & R. Ferreira (3) & & & \\
\hline & H. Helder (3) & & & \\
\hline & L. Neto Jorge (2) & & & \\
\hline & L. Veiga Leitão (6) & & & \\
\hline & Reis (5) & & & \\
\hline PRESENTI 6 AUTORI & 35 AUTORI & 13 AUTORI & 16 AUTORI & 5 AUTORI \\
\hline
\end{tabular}

